\title{
RIGID RINGS
}

\author{
by C. J. MAXSON
}

(Received 27th April 1976)

\section{Introduction}

If $R$ is a ring with identity and $E$ is the semigroup of identity preserving ring endomorphisms of $R$ then the fixed ring of $E$ is defined by $R^{E}=\{a \in R \mid \phi(a)=a$, for each $\phi \in E\} . R$ is said to be rigid if $R^{E}=R$. Equivalently, $R$ is rigid if and only if $E=\left\{\mathrm{id}_{R}\right\}$.

More generally, a ring $R$, not necessarily with an identity, is said to be rigid if $R^{2} \neq(0)$ and the only ring endormorphisms of $R$ are the trivial ones, $0_{R}$ and $\mathrm{id}_{R}$. In this paper we consider the problem of characterising rigid rings.

To provide some setting for the research of this paper we note that fixed rings with respect to certain specialised semigroups have been the objects of study in several investigations. Bergman and Isaacs (1) consider the problem of characterising rings $R$ with the property that $R^{G}=(0)$ where $G$ is a group of automorphisms of $R$. In (5) Herstein investigates rings $R$ being acted upon by a cyclic group $G$ of prime order such that $R^{G}$ is in the center of $R$.

Rigidity in other algebraic structures has also been the topic of previous investigations. In (7), Volpenka, Pultr, and Hedrlin show that there exists a rigid binary relation $\beta$ on any set $X$. Rigid semigroups appear in (3) and rigid rings first appear in (6).

We note that if one drops the restriction " $R^{2} \neq(0)$ " from the definition of rigid rings then the problem of characterising the subclass of rigid rings for which $R^{2}=(0)$ reduces to a well-known result in group theory. That is, a rigid ring $R$ with $R^{2}=(0)$ is just the zero ring on $Z_{2}$.

A brief summary is now given. First some general properties of rigid rings are obtained. In Section 3 chain conditions are imposed. Here we show that a rigid ring $R$ with descending chain conditions on left ideals must be commutative and in several instances $R$ must be a field. In the final section rigid fields are considered.

The author wishes to thank Dr. Matthew J. O'Malley for many helpful suggestions relative to rigid rings.

\section{Properties of Rigid Rings}

In this section we establish some properties of rigid rings and then use these general properties to obtain information about specific classes of rigid rings.

To fix our notation, let $R$ be a ring with Jacobson radical $J(R)$ and centre $Z(R)$. Further let $G(R)$ denote the group of quasi-regular elements of $R$. If $R$ has an identity element then we let $U(R)$ denote the group of units of $R$. 
Our first result establishes that in a rigid ring, $J(R) \subseteq Z(R)$. More generally we have the following.

Theorem 2.1. If $R$ is a rigid ring $G(R) \subseteq Z(R)$.

Proof. Let $a \in G(R)$. Then there exists $b \in R$ such that $0=a+b+a b=$ $a+b+b a$. The map $\phi(a, b): R \rightarrow R$ given by $x \mapsto x+a x+x b+a x b$ is a ring morphism. Consequently $\phi(a, b)=0_{R}$ or $\phi(a, b)=\mathrm{id}_{R}$. If $\phi(a, b)=0_{R}$ then, for every $x$ in $R$, $x+a x+x b+a x b=0$ and in particular $a+a^{2}+a b+a^{2} b=0$. Hence $a=0$ and $a \in$ $Z(R)$. If $\phi(a, b)=\mathrm{id}_{R}$, then, for every $x$ in $R, a x+x b+a x b=0$. Since $b=-a-b a$, $a x+x(-a-b a)+a x(-a-b a)=0$. Thus $a x=x a+(x b+a x+a x b) a$. Hence $a x=x a$ which implies that $a \in Z(R)$.

Corollary 2.1. Let $R$ be a rigid ring. If $R$ is a radical ring then $R$ is commutative.

Suppose $R$ is a rigid ring with an identity element. Then for $u \in U(R)$ the map $\psi: R \rightarrow R$ given by $x \mapsto u x u^{-1}$ is a nonzero ring morphism and thus the identity map. Consequently, we have $U(R) \subseteq Z(R)$.

Let $e$ be a nonzero central idempotent. Then the Peirce decomposition $R=$ $R e \oplus\{r-r e \mid r \in R\}$ is a two-sided decomposition. If $R$ is a rigid ring we must have $R e=R$ and hence $e$ is an identity element for $R$. We now show that every idempotent of a rigid ring is a central idempotent. As a consequence we see that a rigid ring has at most two idempotents.

Theorem 2.2. Every idempotent of a rigid ring is a central idempotent.

Proof. Let $e$ be an idempotent of the rigid ring $R$. Then for each $x$ in $R, e x-e x e$ and $x e-e x e$ are nilpotent and hence quasi-regular. Since $G(R) \subseteq Z(R)$, ex -exe= $e(e x-e x e)=(e x-e x e) e=0=e(x e-e x e)=(x e-e x e) e=x e-e x e$. Thus ex $=x e$ as desired.

We now apply the above properties to show that for many classes of rings the additional hypothesis that a ring of the given class be rigid implies that the ring is commutative.

Theorem 2.3. If $R$ is a rigid ring containing a minimal right ideal $I$ such that $I^{2} \neq(0)$ then $R$ is a field.

(In this paper "field" always means "commutative field".)

Proof. Since $I^{2} \neq(0)$ and $I$ is a minimal right ideal there exists an idempotent $e$ in $I$ such that $I=e R$. But from the above theorem $e$ must be an identity for $R$. Hence, $I=R$. It then follows that $R$ is a division ring and since $R-\{0\}=U(R) \subseteq Z(R), R$ is a field.

Corollary 2.2. If $R$ is a rigid semisimple ring (i.e., $J(R)=(0)$ ) with a minimal right ideal then $R$ is a field. 
For any subring $S$ of a ring $R$, let $[S, S]$ denote the subring of $S$ generated by $\left\{s_{1} s_{2}-s_{2} s_{1} \mid s_{1}, s_{2} \in S\right\}$. For $x \in R$, let $l_{S}(x)=\{s \in S \mid s x=0\}$.

Theorem 2.4. A ring $R$ is commutative if and only if there exists $x \in Z(R)$ such that $R x \subseteq Z(R)$ and $l_{[R, R]}(x)=(0)$.

Proof. Suppose such an element $x$ exists. Let $r, s \in R$. Then $(r s-s r) x=$ $r s x-s r x=s x r-s r x=s r x-s r x=0$. But $r s-s r \in[R, R]$ and $(r s-s r) x=0$ implies $r s-s r=0$. Hence $R$ is commutative. On the other hand if $R$ is commutative, $R=Z(R)$ and $[R, R]=(0)$. Since $R$ has at least one element $x, x \in Z(R), R x \subseteq R=$ $Z(R)$ and $l_{[R, R]}(x) \subseteq[R, R]=(0)$.

Corollary 2.3. Let $R$ be a rigid ring. If there exists some $x \in J(R)$ such that $l_{[R, R]}(x)=(0)$ then $R$ is commutative.

Proof. Since $R$ is rigid, $J(R) \subseteq Z(R)$. Hence $R x \subseteq Z(R)$ and the result now follows from the above theorem.

Of course if $R$ has no divisors of zero then $l_{[R, R]}(x)=(0)$ for each nonzero $x$ in $R$. Thus we can use the above results to obtain information about rigid rings without divisors of zero. In case $R$ is commutative and of prime characteristic a complete characterisation can be given.

Theorem 2.5. Let $D$ be a nonzero commutative ring without divisors of zero. Suppose further that the characteristic of $D$ is a prime, $p$. Then $D$ is a rigid ring if and only if $D \cong Z_{p}$.

Proof. Suppose $D$ is rigid. The map $\phi: D \rightarrow D$ given by $x \mapsto x^{\rho}$ is a nonzero ring morphism. Thus each $x$ in $D$ is a root of the polynomial $f(x)=x^{p}-x$. Let $K$ denote the quotient field of $D$. Then $f(x)$ has at most $p$ roots in $K$. Since every element of $D$ is a root of $f(x)$ in $K, D$ has at most $p$ elements. Thus $D$ is a finite field of cardinality $p$. Consequently $D \cong Z_{p}$. Since the converse is clear, the proof is complete. $F \cong Z_{p}$.

Corollary 2.4. Let $F$ be a field of prime characteristic. $F$ is rigid if and only if

Corollary 2.5. Let $R$ be a nonzero ring without divisors of zero such that $R$ has prime characteristic $p$. If $R$ has a minimal left ideal then $R$ is rigid if and only if $R \cong Z_{p}$.

Proof. Immediate from Theorem 2.3 and Theorem 2.5.

If, in the above corollary, we replace the condition that $R$ have a minimal left ideal by the condition that the centre of $R$ contain a nonzero ideal of $R$ then the same conclusion follows using Corollary 2.3 and Theorem 2.5. We note however, that a characterisation of rigid rings without divisors of zero and of prime characteristic has 
not yet been found. We also mention that the author knows of no example of a noncommutative rigid ring.

\section{Rigid Artinian Rings}

We now restrict our attention to rigid rings satisfying the descending chain condition on left ideals, henceforth called rigid artinian rings. In this case much more can be said. In fact, we find that rigid artinian rings are commutative. Under various hypotheses complete characterisations are obtained.

Theorem 3.1. If $R$ is a rigid artinian ring then $R$ is commutative. If $R \neq J(R)$ then $R$ is a complete local ring. If $R=J(R)$ then $R$ is a nilpotent ring.

Proof. Suppose $R \neq J(R)$. Then $R / J(R)$ is a nonzero semisimple artinian ring and hence $R / J(R)$ has an identity element. Since $J(R)$ is nilpotent, idempotents modulo $J(R)$ can be lifted. Thus $R$ has a nonzero idempotent which must be an identity element for $R$ since $R$ is rigid. Further, since $R$ is artinian with identity and only two idempotents, it is known that $R=U(R) \cup J(R)$. Since $R$ is rigid, $R=U(R) \cup J(R) \subseteq Z(R)$ and thus $R$ is commutative. Since artinian rings with identity are Noetherian, $R$ is a complete local ring.

If $R=J(R)$ then $R$ is a commutative nilpotent ring since $J(R)$ is nilpotent and is contained in $Z(R)$.

In the above proof we also established the following.

Corollary 3.1. Let $R$ be a rigid artinian ring. The following are equivalent:

(i) $R$ has an identity,

(ii) $R \neq J(R)$,

(iii) $R$ is a complete local ring.

As a consequence we have that a finite rigid ring with identity is a complete local ring. We present an example to show that this does not characterise finite rigid rings with identity.

Example. Let $H \equiv\langle H,+\rangle$ be the group $\left\langle Z_{3} \oplus Z_{3},+\right\rangle$ and define a multiplication $*$ on $H$ by $(a, b) *(c, d)=(a c, a d+b c)$. Then $\langle H,+, *\rangle$ is a complete local ring with identity. However, the map $\phi: H \rightarrow H$ given by $(a, b) \mapsto(a, 0)$ is a nonzero, nonidentity ring morphism.

In order to further classify rigid artinian rings with identity we apply some known results concerning the structure of complete local rings. As is often the case in the study of local rings, the "equal characteristics" case is easier to handle than the "unequal characteristics" case.

Theorem 3.2. If $R$ is a rigid artinian ring with identity and with prime or zero characteristic then $R$ is a field.

Proof. We first show that the characteristic of the complete local ring $R$ is the same as the characteristic of its residue field $R / J(R)$. 
Suppose $\operatorname{ch}(R / J(R))=0$. Thus, if 1 denotes the identity of $R, n \cdot 1 \notin J(R)$ for each positive integer $n$. Consequently, $n \cdot 1 \neq 0$ for each positive integer $n$. Hence $\operatorname{ch}(R)=0$.

Suppose $\operatorname{ch}(R / J(R))=p$ where $p$ is a prime. Then $p \cdot 1 \in J(R)$. Since $J(R)$ is nilpotent there exists a positive integer $t$ such that $0=(p \cdot 1)^{t}=p^{t} \cdot 1$. This implies that the characteristic of $R$ equals $p^{s}$ for some positive integer $s, 1 \leqslant s \leqslant t$. Since, by hypothesis the characteristic of $R$ is either zero or a prime, we have $\operatorname{ch}(R)=p$.

Thus $R$ is a complete local ring with the same characteristic as its residue field $R / J(R)$. Hence (2, Theorem 9, p. 72) $R$ contains a subfield $K$ isomorphic to $R / J(R)$. Let $\psi: R / J(R) \rightarrow K$ be an isomorphism, let $\phi: R \rightarrow R / J(R)$ be the canonical epimorphism and let $i: K \rightarrow R$ be the insertion morphism. Then $i \psi \phi=\mathrm{id}_{R}$. Hence $R$ is a field.

Corollary 3.2. Let $R$ be an artinian ring with identity and of prime characteristic. $R$ is rigid if and only if $R \cong Z_{p}$.

Proof. This is immediate from the above theorem and Corollary 2.4 .

Turning to the unequal characteristic case we have only a partial characterisation. For this we suppose $R$ is a rigid artinian ring with identity such that $\operatorname{ch}(R)=p^{k}, k>1$, and $\operatorname{ch}(R / J(R))=p$ where $p$ is a prime. Suppose further that $J(R)$ is generated by $p \cdot 1$; i.e., $J(R)=R \cdot p$. Thus by $(2$, Corollary 3, p. 83$), R \cong C /\left((p \cdot 1)^{k}\right)$ where $C$ is a complete discrete unramified valuation ring of characteristic zero and such that $C / J(C) \cong R / J(R)$.

If $R / J(R)$ is a perfect field then $C$ can be taken as the ring of Witt vectors over $R / J(R)$ and hence $R\left(=C /\left((p \cdot 1)^{k}\right)\right.$ can be considered as the ring of Witt vectors over $R / J(R)$ of length $k$. (See (4), Chapter 6 for results on Witt vectors.) Now, the map $\phi: C /\left((p \cdot 1)^{k}\right) \rightarrow C /\left((p \cdot 1)^{k}\right)$ given by $\left(a_{0}, a_{1}, \ldots, a_{R-1}\right) \mapsto\left(a_{0}^{p}, a_{1}^{p}, \ldots, a_{R-1}^{p}\right)$ is a nonzero ring morphism (see (4)) and since $R$ is rigid this implies that $a_{0}^{p}=a_{0}$ for all $a_{0} \in$ $R / J(R)$. Hence $R / J(R) \cong Z_{p}$. Thus the ring of Witt vectors over $R / J(R)$ of length $k$ has $p^{k}$ elements. Since $Z_{p^{k}}$ can be embedded in $C /\left((p \cdot 1)^{k}\right)$, we have $Z_{p^{k}} \cong R$.

We have established the following.

Theorem 3.3. Let $R$ be a rigid artinian ring with identity such that $\operatorname{ch}(R)=p^{k}$, $k>1, p$ a prime and $J(R)=R \cdot p$. If $R / J(R)$ is a perfect field then $R \cong Z_{p^{k}}$. Conversely, if $R \cong Z_{p^{k}}, k>1, p$ a prime, then $R$ is a rigid artinian ring with identity, $J(R)=R \cdot p$ and $R / J(R)$ is a perfect field.

\section{Rigid Fields}

In Theorem 3.2 we found that a rigid artinian ring with identity of prime or zero characteristic is a field. Further, in Corollary 2.4 , rigid fields of prime characteristic were characterised. In this section we give a few remarks about rigid fields of zero characteristic.

We say that a subring $R$ of a ring $S$ is $S$-rigid if for every endomorphism $\phi$ of $S$, $\phi \mid R=0_{R}$ or $\phi \mid R=\mathrm{id}_{R}$. Clearly every subring of a rigid ring $S$ is $S$-rigid. Moreover, if $P$ is a prime field then $P$ is $K$-rigid for every extension $K$ of $P$. 
Theorem 4.1. Let $K$ be a field such that for some $K$-rigid subfield $A$ of $K$, $K=A(\alpha) . K$ is rigid if and only if the minimum polynomial, $p_{\alpha}(x)$, of $\alpha$ over $A$ has exactly one root in $K$.

Proof. For every $\beta$ in $K$ such that $p_{\alpha}(\beta)=0$, the map leaving $A$ fixed and taking $\alpha$ into $\beta$ determines an endormorphism of $K$. Consequently, if $K$ is rigid, $\alpha$ must be the only root of $p_{\alpha}(x)$ in $K$. On the other hand, let $\phi$ be a non-zero element in End $(K)$ and let $\beta=\phi(\alpha)$. Since $\phi \mid A=1_{A}, p_{\alpha}(\beta)=0$. Hence, $\alpha=\beta$ which in turn implies that $\phi$ is the identity on $K$.

As an application of the above theorem we note that $Q(\sqrt[n]{p})$ is rigid for all primes $p$ and all odd positive integers $n$, where $Q$ denotes the field of rational numbers.

More generally, if $K$ is a finite algebraic extension of some $K$-rigid subfield $A$ then, from the "theorem of primitive elements", $K=A(\alpha)$. Hence the above theorem gives necessary and sufficient conditions for a finite algebraic extension of the rationals to be rigid. In our next theorem we consider arbitrary algebraic extensions.

Lemma 4.1. If $K$ is an algebraic extension of a $K$-rigid field $A$, then every nonzero endomorphism of $K$ is an automorphism.

Proof. Since every nonzero endomorphism is one-one it remains to show that every nonzero endomorphism is an onto map. Let $\phi$ be a nonzero endomorphism of $K$ and let $a$ be an arbitrary element in $K$. Now $a$ is a root of an irreducible polynomial $f(x) \in A[x]$. Let $a=a_{0}, a_{1}, \ldots, a_{t}$ be the distinct roots of $f(x)$ in $K$. Since $\phi \mid A=\operatorname{id}_{A}$, $\phi\left(a_{0}\right), \phi\left(a_{1}\right), \ldots, \phi\left(a_{t}\right)$ are also distinct roots of $f(x)$ in $K$. Hence for some $a_{i}$, $\phi\left(a_{i}\right)=a$. Hence $\phi$ is an automorphism of $K$.

Recall that a field $K$ is a normal extension of a field $A$ if the fixed field of $\operatorname{Aut}(K, A)$ is $A$; i.e., $K^{\operatorname{Aut}(K, A)}=A$.

Theorem 4.2 Let $K$ be an algebraic extension of some $K$-rigid subfield $A . K$ is rigid if and only if $K$ is not a normal extension of any of its proper subfields.

Proof. Suppose $K$ is a rigid field and $H$ a proper subfield of $K$. Thus $K^{\text {Aut }(K, H)}=$ $K \neq H$. Hence, $K$ is not a normal extension of $K$. Conversely suppose $K$ is not rigid. Then by the above lemma there is a nonzero automorphism $\psi$ of $K$ such that $\psi \neq$ id $_{K}$. Let $K^{\psi}=\{a \in K \mid \psi(a)=a\}$. Since $\psi \neq \mathrm{id}_{K}, K^{\psi} \subset K$. Now let $G=\operatorname{Aut}\left(K, K^{\psi}\right)$. Since $\psi \in G, K^{G} \subseteq K^{\psi}$. Clearly $K^{\psi} \subseteq K^{G}$. Thus $K^{G}=K^{\psi}$ and consequently $K$ is a normal extension of $K^{\psi}$.

Since the rational numbers $Q$ are $K$-rigid for every extension $K$ of $Q$ the above theorem gives necessary and sufficient conditions for an algebraic extension of $Q$ to be rigid.

\section{REFERENCES}

(1) G. M. Bergman and I. M. Is AACS, Rings with fixed point-free group actions, Proc. London Math. Soc. (3), 27 (1973), 69-87. 
(2) I. S. CoHEn, On the structure and ideal theory of complete local rings, Trans. Amer. Math. Soc. 59 (1946), 54-106.

(3) V. Dlab and B. H. NeumanN, Semigroups with few endomorphisms, J. Aust. Math. Soc. 10 (1969), 162-168.

(4) M. GREenberg, Lectures on Forms in Many Variables (W. A. Benjamin, New York, 1969).

(5) I. N. HERSTEIN, Rings admitting certain automorphisms, Istanbul Üniv. Fen Fak. Mec. Ser. A 26 (1961), 45-59.

(6) C. J. MAXSON, $(0,1)$-matrices and semigroups of ring endormorphisms, Semigroup Forum 5 (1972), 160-166.

(7) P. Volpenka, A. Pultr and Z. Hedrlin, A rigid relation exists on any set, Comment. Math. Univ. Carolinae 6 (1965), 149-155.

TEXAS A \& M UNIVERSITY,

College Station, Texas 77843 